\title{
Perioral Aerosol Sequestration Suction Device Effectively Reduces Biological Cross-Contamination in Dental Procedures
}

\author{
Víctor Lloro ${ }^{1, \odot ~ M a r i a ~ L a u r a ~ G i o v a n n o n i 2 ~}{ }^{2, \odot} \quad$ Vicente Lozano-de Luaces $^{3} \quad$ Maria Cristina Manzanares ${ }^{1, \odot}$ \\ ${ }^{1}$ Human Anatomy and Embryology Unit, Experimental Pathology \\ and Therapeutics Department, Faculty of Medicine and Health \\ Sciences, Health University of Barcelona, Barcelona, Spain \\ ${ }^{2}$ Odontology Hospital UB, Odontostomatology Department, Faculty \\ of Medicine and Health Sciences, Health University of Barcelona, \\ Barcelona, Spain \\ ${ }^{3}$ Faculty of Medicine and Health Sciences, Health University of \\ Barcelona, Barcelona, Spain \\ Eur ] Dent 2021;15:340-346 \\ Address for correspondence Víctor Lloro, DDS, MSc, \\ 5305, P Govern, Health University of Barcelona Campus, \\ Feixa Llarga Street, L'Hospitalet, 08907 Barcelona, Spain \\ (e-mail: vllorobo7@alumnes.ub.edu).
}

\begin{abstract}
Keywords

- aerosol

- ATP luminescent measurements

- cross-transmission

- infection disease transmission

- oral health

- SARS-CoV-2
\end{abstract}

Objective The infection risk during dental procedures is a common concern for dental professionals which has increased due to coronavirus (severe acute respiratory syndrome coronavirus 2) pandemic. The development of devices to specifically mitigate cross-contamination by droplet/splatter is crucial to stop infection transmission. The objective of this study is to assess the effectiveness of a perioral suction device (Oral BioFilter, OBF) to reduce biological contamination spread during dental procedures.

Materials and Methods Forty patients were randomized 1:1 to a standard professional dental hygiene treatment with OBF and without. Adenosine triphosphate (ATP) bioluminescence assay was used to evaluate the spread of potential contaminants. The total number of relative light units (RLU) from key dental operatory locations: operator's face-shield, back of the surgical operator's-gloves, patient's safety-goggles, and instrumental table were measured. Percentage contamination reductions between control and OBF were compared.

Statistical Analysis Primary outcome, total RLU, was analyzed by comparing the means of logged data, using a two-sided two-sample $t$-test. Secondary outcomes as RLU of logged data for the different locations were analyzed in the same way. Proportion of patients from whom different locations reported events (clean, acceptable, and failure) were analyzed by using Fisher's exact test.

Results For the whole dental environment, RLUs reduction ( $<150$ units) achieved with OBF was $98.4 \%$ (97.4-99\%). By dental operatory location the reduction in RLUs published online March 12, 2021
Dol https://doi.org/

10.1055/s-0041-1724152 ISSN 1305-7456. (c) 2021. European Journal of Dentistry.

This is an open access article published by Thieme under the terms of the Creative Commons Attribution License, permitting unrestricted use, distribution, and reproduction so long as the original work is properly cited. (https://creativecommons. org/licenses/by/4.0/)

Thieme Medical and Scientific Publishers Pvt. Ltd. A-12, 2nd Floor, Sector 2, Noida-201301 UP, India 
was from $99.6 \%$, on the operator face-shield, to $83 \%$ on instrumental table. The control group reported a very high percentage of failures, $(>300)$ being $100 \%$ on the surfaces closer to the patient's mouth and decreasing to $70 \%$ on instrumental table. In contrast, the higher failure percentage in the OBF group was found on the patient's goggles (40\%), while the operator face-shield showed an absence of contamination.

Conclusion OBF device has shown efficient reduction of biological aerosol cross-contamination during dental procedures as proved by ATP-bioluminescence assay. Nevertheless, for maximum safety, its use must be combined with standard protective gear such as goggles, face shield, and surgical gloves.

\section{Introduction}

The cross-contamination and dissemination of pathogens in a dental operatory environment is a concern during dental procedures. The recent pandemic caused by coronavirus (SARS-CoV-2) that on February 11, 2020, World Health Organization named as "coronavirus disease" 1 has increased this concern. Dental professionals must be aware that droplet and splatter have proven to be the main spread routes of oral pathogens and, among them, SARS-CoV-2. ${ }^{1}$

Oral droplet and splatter are biological substances (made of a combination of particles, gases, vapors, biological fragments, or microorganisms that are or become airborne) with a diameter between 0.5 and $10 \mu \mathrm{m}$ that have the potential to penetrate and lodge in the smaller passages of the lungs., ${ }^{2,3}$ These particles are disseminated during standard dental treatments (e.g., ultrasonic scaling, professional mechanical tooth cleaning, etc.) and are potential sources of cross-transmission of microorganisms. ${ }^{4}$ In this regard, a recent study has shown that instruments, such as rotating tools, ultrasonic scalers, and piezo tools, produce a greater amount of aerosols/spray during dental procedures that can widely spread the saliva of a potentially infected patient, thereby exponentially increasing the risk of contamination/contagion. ${ }^{5}$

These considerations have gained relevance since SARS-CoV-2 represents a high risk for cross-transmission of microorganisms for oral health professionals (OHP), which can become potential carriers of the disease due to the unique nature of oral health procedures, that involve aerosol generation and the close proximity of the professionals to the highly contaminated oropharyngeal region of symptomatic and asymptomatic patients. ${ }^{6}$ Besides, as reported by Zemouri et al, ${ }^{7}$ patients could also generate droplets and splatter by coughing, sneezing, and talking; therefore, the transmission probability is driven by indoor air quality, patients infectiousness, and the masks used by OHPs. Actually, Gou et al concluded that SARS$\mathrm{CoV}-2$ has been widely distributed in the air (as an aerosol) and on object surfaces with transmission distances close to $4 \mathrm{~m}$ in hospital facilities such as intensive care units. This poses a high infection risk for medical staff and other close persons. Most particularly, such risk is the highest in small indoor rooms. ${ }^{8}$
Measuring the concentration of adenosine triphosphate (ATP) by bioluminescence assay is a technique routinely used in hospitals and the food industry ${ }^{9-12}$ for fast and accurate quantification of biological contamination on surfaces. ATP systems are able to detect dried organic materials and dead microorganisms ${ }^{9}$ and to determine the efficacy of environmental cleanliness procedures even with low microbial counts, ${ }^{13,14}$ but do not detect viruses. This measurement technique has been successfully used in a recent dentistry study which showed increased biocontamination levels on operator's goggles, masks, arms, and patient's goggles after standard dental procedures, demonstrating the ability of generated droplet and splatter to spread infection among operators and patients. ${ }^{4}$

Cross-transmission of microorganisms can be prevented by preventing the contamination. ${ }^{15}$ This can be achieved either at a macro level increasing ventilation or at the source of contamination by applying an aerosol suction device, like the OBF, which was initially developed for astronauts in space. In the absence of gravity, particles of saliva or blood containing pathogens are released into the environment ${ }^{16,17}$ as also reported in patients in isolated missions ${ }^{17}$ both probably due to reduced ventilation, as suggested by Zemouri et al, ${ }^{7}$ which studied the analogous situation in dental clinics due to the indoor air quality. The perioral suction device named Oral Biofilter (OBF) consists in an ergonomic lip retractor that supports an extraoral suction device which connects to the dental chair's high-volume evacuator (HVE) suction system. This device acts as a filter that prevents airborne microparticles from expanding beyond the work area, thus harming the OHP as well as the patients. As a consequence, OBF improves everyone's safety by keeping the dental workplace hygiene and cleanliness. ${ }^{16,18}$

The aim of the present study is to assess the effectiveness of the OBF device to reduce cross-transmission of microorganisms by analyzing the contamination patterns produced by droplet and splatter during professional dental hygiene with ultrasonic scaling, followed by professional mechanical tooth cleaning (PMTC) with high-speed contra-angle handpiece. 


\section{Materials and Methods}

\section{Study Design}

The study was performed during the routine clinical practice in patients undergoing a programmed professional dental hygiene with ultrasonic scaler followed by a PMTC. All participants gave written informed consent to the procedure, which was approved by the University of Barcelona Bioethics Committee, as a part of the first author $\mathrm{PhD}$ Thesis project, and was conducted in accordance with the Helsinki Declaration of 1975, as revised in 2013.

The standard professional dental hygiene procedure was performed by a single right-handed hygienist at a height of $1.55 \mathrm{~m}$ with no assistant, using an ultrasonic scaler (Satelec Acteon group, Merignac, France) during $10 \mathrm{~min}-$ utes, followed by polishing with polishing paste and NSK contra-angle (M 25 S-MAX, NSK, Nakanishi, Tochigi-ken, Japan). As a pretreatment standard procedure, patients' oral cavity was rinsed with $0.2 \%$ povidone iodine for 1 minute to reduce pathogen load stemming from droplet and splatter forming procedures in dental settings. ${ }^{19,20}$ The instrumental table was located $1 \mathrm{~m}$ from the patient's mouth, on the right of the patient and in front of the hygienist. Patients were lying down in the dentist' chair (FEDESA JS 500, FEDESA, Madrid, Spain), with a distance between the operator's face shield and the patient's mouth of $30 \mathrm{~cm}$ ( - Fig. 1). The procedure was carried out with a dental chair's HVE suction system, which provided at the HVE hose while saliva ejector was also active a relative negative pressure of 143 mbar measured by a vacuum meter (Thyracont VD81, Thyracont, Passau, Germany) and an air flow of 240 liters per minute (DURR ROTA 84.4000 SW N4 10× flowmeter, Dürr systems, Bietigheim-Bissingen, Germany). The sole difference between the control and the OBF group was the static device connecting the HVE system with the patient's mouth, during a dental hygiene two hands procedure, with the OHP keeping the intraoral mirror in the left hand while scaling with the right hand.

Patients were randomized 1:1 to use or not the OBF device. The study primary outcome was the total number of RLU from every location of dental environment: operator's face shield, operator's back of the surgical glove, patient's safety goggles, and instrumental table. Secondary outcomes were percentage contamination differences at the different locations between control and OBF groups.
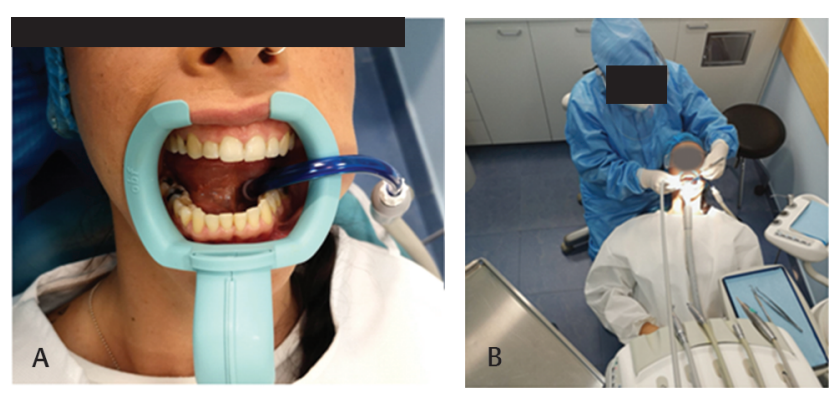

Fig. 1 (A) Image of Oral BioFilter device. (B) Representative image of professional hygienic procedure.

\section{Sample Collection and Illuminometer Assay}

The analyzed surfaces were the instrumental table, the operator's right hand protection (long cuff sterile latex surgical gloves), the patient's safety goggles, and the operator's face shield. They were cleaned with $70 \%$ alcohol disposable gauze just before the dental procedure and after taking the samples. Operator's gloves were changed and discarded between patients.

Samples were obtained immediately after dental procedure finalization from the aforementioned surfaces from the patient as well as the operator using a chemically impregnated reagent cotton swab (ATP Test Swab UXL100, 3M, Minnesota, United States) for ATP assay. Areas measured consisted in $100 \mathrm{~cm}^{2}$ on the surface of the face shield, patient dental goggles, OHP back of right-hand glove, and instrumental table.

All samples were obtained by a second operator, also provided with the adequate protective equipment. The ATP bioluminescence assessment was performed as described by Sanna et al., ${ }^{11}$ by using a Lumitester (3M Clean-Trace LM1, 3M, Minnesota, United States) according to the manufacturer's instructions.

Biocontamination levels, expressed in relative light units (RLUs) which in turn are a function of ATP concentration, were recorded. An RLU level $<150$ units was considered as clean; RLU levels ranging from 151 to 300 were considered as acceptable, and an RLU level $>300$ was considered as a failure of the cleaning protocol, a threshold based on the report by Ho et $\mathrm{al}^{21}$ as well as the manufacturer's recommendations.

\section{Statistical Analysis}

Variables were described by using summary statistics as median, minimum, and maximum values and splatted by group (OBF and control). The Shapiro-Wilk test was used to check normality of transformed data.

The potentially contaminated areas $\left(100 \mathrm{~cm}^{2}\right)$ were measured on the surface of the operator's face shield and back of the right-hand glove, the patient's goggles, and the instrumental table. After the natural logarithm transformation, a centered distribution was reached (ShapiroWilk $p=0.5447$ for OBF and $p=0.8553$ for control groups).

Primary outcome, total RLU, was analyzed by comparing the means of logged data, using a two-sided two-sample $t$-test. Conclusions were drawn in terms of percentage of reduction as ratio of $(G M-1) \times 100$, where $G M$ was the geometric mean and their confidence intervals.

Power calculation was performed taking into account a previous pilot study (data not shown), in which a coefficient of variation on the original scale of 12 was obtained, then a two-sided two-sample $t$-test with 20 subjects per group and significance level of 0.05 achieved $94 \%$ power to detect a ratio of geometric means of 0.08 or reduction of $92 \%$ when using OBF.

Secondary outcomes as RLU of logged data for the different locations were analyzed in the same way. Proportion of patients from whom different locations reported events (clean, acceptable, and failure) were analyzed by using 
Fisher's exact test. A natural logarithm transformation was applied for total RLUs and for every location due to the extremely asymmetrical distribution of data.

Randomization list and power calculation were performed with PASS 2020 (PASS 2020 Power Analysis and Sample Size Software (2020). NCSS, LLC. Kaysville, Utah, USA css.com/software/pass) and data management and statistical analysis with STATA 16 (StataCorp. 2019. Stata Statistical Software: Release 16. College Station, Texas, United States: StataCorp LLC).

\section{Results}

The study was carried out on May and June 2020. In total, 40 patients were included 16 males and 24 females, with a mean age (standard deviation) of 45.6 (16.4) years and 40.6 (13.7) years, respectively.

The mean RLU values and the difference between groups (total and by each location) are shown in - Table 1, indicating the percentage of reduction using OBF with respect to control. For the whole dental environment, the percentage of reduction in RLUs achieved with OBF was $98.4 \%$; this reduction ranged from 97.4 to $99 \%$ in the $95 \%$ of the cases (-Table 1). Regarding the analysis by locations, the use of OBF showed different percentages of reduction in RLUs ranging from the maximum, a 99.6\%, in the OHP face shield, to a minimum (83\%) in the instrumental table (-Table 1).

Regarding the three cleaning levels according to the RLUs values, high differences were also found favoring OBF group in all measured locations (-Table 2). The control group reported a very high percentage of failures, being the $100 \%$ in the surfaces nearer to the patient's mouth and decreasing

Table 1 Comparison between groups for total relative light unit and by locations for logged data

\begin{tabular}{|c|c|c|c|c|c|}
\hline Location & Mean & Difference & $\begin{array}{l}\text { Reduction } \\
(\%)^{\mathrm{a}}\end{array}$ & $95 \% \mathrm{Cl}$ & $p$-Value \\
\hline \multicolumn{6}{|l|}{ All } \\
\hline OBF & 6.62 & -4.15 & 98.4 & $97.4-99.0$ & $<0.001$ \\
\hline Control & 10.77 & & & & \\
\hline \multicolumn{6}{|l|}{ Face shield } \\
\hline $\mathrm{OBF}$ & 4.33 & -5.46 & 99.6 & $99.2-99.8$ & $<0.001$ \\
\hline Control & 9.79 & & & & \\
\hline \multicolumn{6}{|c|}{ Surgical glove } \\
\hline $\mathrm{OBF}$ & 5.31 & -3.69 & 97.5 & $94.7-98.8$ & $<0.001$ \\
\hline Control & 9.00 & & & & \\
\hline \multicolumn{6}{|c|}{ Patient safety goggles } \\
\hline OBF & 5.63 & -2.76 & 93.7 & $86.3-97.1$ & $<0.001$ \\
\hline Control & 8.39 & & & & \\
\hline \multicolumn{6}{|c|}{ Instrumental table } \\
\hline OBF & 4.42 & -1.77 & 83.0 & $66.1-91.6$ & $<0.001$ \\
\hline Control & 6.19 & & & & \\
\hline
\end{tabular}

Abbreviations: $\mathrm{Cl}$, confidence interval; $\mathrm{GM}$, geometric means; OBF, oral biofilter.

Reduction: (ratio of GM - 1) × 100 . to $70 \%$ in the instrumental table. In contrast, the higher percentage of failures in the OBF group was found in the patient's goggles (40\%), while the OHP face shield showed a complete absence of particles as revealed by RLU counts.

The results of the present study are consistent in proving that the use OBF device reduces the bioluminescent droplet and splatter particles and splatter produced by dental procedures. The grouped results showed a $98.4 \%$ of whole dental environment contaminant reduction.

\section{Discussion}

In general dental practice, the use of face seal masks was recommended to reduce the exposure to aerosolized microorganisms such as Mycobacterium tuberculosis. ${ }^{22}$ Influenza or SARS-CoV virus may be also shed into the environment and environmental surfaces and transferred to hands of patients and health care providers ${ }^{23}$ as well as form airborne particles.,7 The pandemic has made more evident the need for masks since SARS-CoV-2 has been consistently detected in the saliva of COVID-19 patients. ${ }^{4,24,25}$ In two recent reports, Zemouri et $\mathrm{al}^{7,12}$ have reported that the highest transmission possibilities estimated in a dental clinic were for measles virus (100\%), followed by coronaviruses (99.4\%), influenza virus (89.4\%), and M. tuberculosis (84\%).

The detection of biological contamination in dental clinics provides useful information to promote directed hygienic measures and avoid biohazard transmission. The ATP levels (ATP bioluminescence assay) detected on surfaces and equipment showed a positive correlation with bacterial contamination in dental practice. ${ }^{12}$ Bioluminescence has not proven to date a direct correlation between viable microbial counts and detected ATP levels., ${ }^{9,13}$ However, ATP-bioluminescence is a valuable tool for determining the efficiency of cleanliness procedures as well as to distinguish between live and dead cells, ${ }^{13}$ but it is not efficient in detection environmental, not cell-bound ATP and viruses.

Using ATP bioluminescence, Watanabe et al reported significant higher values of contamination and splatters in surfaces produced by droplet and splatter in operator masks, goggles, chest, and gowned right arm after dental procedures. ${ }^{4}$ Zemouri et $\mathrm{al}^{7,12}$ estimated that, even if the contamination due to droplet and splatter may be low in a dental clinic, the highest level of contaminated particles is to be found within $80 \mathrm{~cm}$ around the head of the patient because the droplet and splatter produced by oral health treatments may contain a similar number of pathogens as compared with coughing and sneezing. Although the exact numbers of viral pathogens in droplet and splatter are not known to date, Zemouri et $\mathrm{al}^{7}$ as well as Prather et $\mathrm{al}^{26}$ suggest that SARS-CoV-2 is spreading in droplet and splatter exhaled by highly contagious, nonsymptomatic individuals.

It is important to remark that although our study has focused in assessing the novel OBF device efficacy for cross-contamination reduction; personal protective equipment such as face shield, mask, goggles, and surgical gloves; and protective measures are still fundamental elements to mitigate infectious risks. Several studies have shown how 
Table 2 Counts of occasions with clean, acceptable, and failure events by locations

\begin{tabular}{|c|c|c|c|c|}
\hline Location & $\begin{array}{l}\text { Clean } \\
(\text { RLU < 150) }\end{array}$ & $\begin{array}{l}\text { Acceptable } \\
(\text { RLU = 150-300) }\end{array}$ & $\begin{array}{l}\text { Failure } \\
(\text { RLU > 300) }\end{array}$ & $p$-Value \\
\hline \multicolumn{5}{|c|}{ Face shield } \\
\hline OBF & $15(75)$ & $5(25)$ & 0 & $<0.001$ \\
\hline Control & 0 & 0 & $20(100)$ & \\
\hline \multicolumn{5}{|c|}{ Surgical glove } \\
\hline OBF & $8(40)$ & $5(25)$ & $7(35)$ & $<0.001$ \\
\hline Control & 0 & 0 & $20(100)$ & \\
\hline \multicolumn{5}{|c|}{ Patient safety goggles } \\
\hline OBF & $6(30)$ & $6(30)$ & $8(40)$ & $<0.001$ \\
\hline Control & $1(5)$ & 0 & $19(95)$ & \\
\hline \multicolumn{5}{|c|}{ Instrumental table } \\
\hline OBF & $15(75)$ & $4(20)$ & $1(5)$ & $<0.001$ \\
\hline Control & $4(20)$ & $2(10)$ & $14(70)$ & \\
\hline
\end{tabular}

Abbreviations: OBF, oral biofilter; RLU, relative light unit.

Note: Data are expressed as counts (\%).

such protective gear coupled with a reasonable dental equipment use are important to prevent infections. ${ }^{27,28}$

In our study, the impact of using a OBF device was assessed by using the same biological detection assay used by Watanabe et $\mathrm{al}^{4}{ }^{4}$ in patients after a standard dental hygiene procedure. Since patients both groups of patients were submitted to the same standard procedure that includes a $0.2 \%$ povidone iodine mouthwash for 1 minute, the ATP bioluminescence measurement was aimed to detect and localize the biological cross-contamination, be that constituted by living or dead cells and particles. We observed a significant decrease, around $98.4 \%$, in whole dental environment contamination with the use of OBF. Our results corroborate the risk of biological cross-contamination by droplet and splatter described by Watanabe et $\mathrm{al}^{4}$ and Zemouri et $\mathrm{al}^{7,12}$ in operator's and patient's protective gear, thus demonstrating the utility of bioluminescence to assess the reduction of droplet and splatter spreading by using OBF. The limitation of our study is that bioluminescence, despite being able to identify living microorganisms, such as bacteria, dinoflagellates, and fungi, does not detect the presence of viruses. ${ }^{13}$

Despite reports of measurable droplet and splatter reduction have encouraged the HVE use in routine dental practice while working with four hands, ${ }^{29}$ other authors did not find neither significant droplet and splatter counts decrease nor environmental contamination reductions unless the HVE aspirator was subject to modifications. ${ }^{30}$ In our study, the OBF device as provided by the manufacturer was attached to the standard HVE hose while permitting the OHP to operate with two hands without assistant. A much improved reduction of droplet and splatter dissemination was evidenced, when compared with the control group, that do not used the HVE device because is incompatible with the use of an intraoral mirror in two hands work. Moreover, we wanted to corroborate the dispersion distance of particles Watanabe et al found in their study ${ }^{4}$ and to analyze the effectiveness of the
OBF device against the total spread of droplet and splatter particles. For this reason, further analysis of the droplet and splatter reduction in four hands work, where the operator's dental assistant will handle the HVE and the saliva ejector, while the operator will continue to use ultrasound and the intraoral mirror are planned.

Watanabe et al reported that the surface with the highest contamination levels was in the patient's safety goggles, ${ }^{4}$ while Zemouri et al found the highest bacterial contamination on the patient's chest area. ${ }^{12}$ Previous studies highlighted that eye infections are common among dentists, ${ }^{31}$ which risk being also signaled for SARS-CoV-2 both for general population ${ }^{32}$ and for dentists. Moreover, blood or saliva contained in the splatters could produce infection and corneal epithelial exfoliation, conjunctivitis, and keratitis. ${ }^{33}$ With the use of OBF, the droplet and splatter reduction achieved $97.3 \%$ in patient's goggles and $99.6 \%$ in OHP's face shield, thus providing an extra protective measure against ocular cross-contamination.

The OHP's hand is the surface closest to patient's mouth; therefore, a contamination of this surface is common in clinical practice. The use of OBF promotes a reduction of contamination on the surgical gloves of $97.5 \%$ versus control group (without $\mathrm{OBF}$ ). This result demonstrates that OBF could also enhance the prevention of biological cross-contamination in high-risk airway management procedures ${ }^{34}$ as well as in orofacial aerosol-generating procedures also usual in head and neck surgery, ${ }^{35}$ otolaryngology, ${ }^{36}$ and anesthesia. ${ }^{37}$ Although this noticeable decrease represents an improvement of the cross-contamination control, for safety purposes, adopting updated guidelines on infection prevention ${ }^{7,12,38,39}$ is still mandatory.

It is interesting to note that the lowest percentage of the contamination reduction achieved with the OBF device was found in the instrumental table ( $83 \%$ reduction of biological contamination) located at a distance of $1 \mathrm{~m}$ from the patient which agrees with the $80 \mathrm{~cm}$ distance 
reported by Zemouri et al. ${ }^{7}$ At first glance, despite being statistically significant, such reduction highlights the difference between $70 \%$ unacceptably contaminated surface cases (control group) down and just 1\% (OBF group; $p<$ 0.001 ). On the other hand, particles sized between 1 and 4 microns expelled by patients just by breathing have the potential of lingering in the air ${ }^{7,26}$ and may be displaced by airflows and deposited on equipment. ${ }^{40}$ This result is relevant as the material placed on the instrument table should be preserved in the cleanest possible condition after being unpacked, so further measures have to be taken to avoid cross contamination on this area.

The significant difference among RLU measures between both groups (OBF vs. control; 6.62 vs. 10.77 [difference = -4.15 ]; $p$ <.001) confirms that the major effect of OBF is for the OHPs. When proper infection control measures are used, the chance for the patient to become contaminated by microorganisms from the previous patient is substantially limited. Therefore, only in the event that these standard measures failed due to human error, we can consider that the device adds extra protection to the next patient. The device does not remove the infection risk but reduces the cross-contamination risk by limiting the droplet and splatters.

Taken together, these first results show a significant reduction of cross-contamination on oral health operatory surfaces by using the OBF device as well as relevant information about which specific surfaces require additional hygienic measures to ensure patient's and OHPs protection.

In conclusion, to our knowledge, this is the first study that uses a specific device designed to reduce the biological cross-contamination in routine oral health practice. It is important to remark that OBF device provides a much improved protection, but it is no substitute to other protective measures such as face shield and mask, goggles, and surgical gloves which must still be considered mandatory.

\section{Funding}

The study obtained its funding from University of Barcelona Faculty of Medicine and Health Sciences Barcelona, Catalunya, ES.

\section{Conflict of Interest}

None declared.

\section{Acknowledgements}

The authors gratefully acknowledge the collaboration of ASTRADENTIUM Health Technologies and 3M Company for the loan of, respectively, OBF devices and the Lumitester device.

\section{References}

1 Peng X, Xu X, Li Y, Cheng L, Zhou X, Ren B. Transmission routes of 2019-nCoV and controls in dental practice. Int J Oral Sci 2020; 12(1):9

2 Harrel SK, Molinari J. Aerosols and splatter in dentistry: a brief review of the literature and infection control implications. J Am Dent Assoc 2004; 135(4):429-437

3 Kumar PS, Subramanian K. Demystifying the mist: sources of microbial bioload in dental aerosols. J Periodontol 2020; 91(9):1113-1122
4 Watanabe A, Tamaki N, Yokota K, Matsuyama M, Kokeguchi $\mathrm{S}$. Use of ATP bioluminescence to survey the spread of aerosol and splatter during dental treatments. J Hosp Infect 2018; 99(3):303-305

5 Bizzoca ME, Campisi G, Lo Muzio L. An innovative risk-scoring system of dental procedures and safety protocols in the COVID-19 era. BMC Oral Health 2020; 20(1):301

6 Ather A, Patel B, Ruparel NB, Diogenes A, Hargreaves KM. Coronavirus disease 19 (COVID-19): implications for clinical dental care. J Endod 2020; 46(5):584-595

7 Zemouri C, Awad SF,Volgenant CMC, Crielaard W, Laheij AMGA, de Soet JJ. Modeling of the transmission of coronaviruses, measles virus, influenza virus, Mycobacterium tuberculosis, and Legionella pneumophila in dental clinics. J Dent Res 2020; 99 (10):1192-1198

8 Guo ZD, Wang ZY, Zhang SF, et al. Aerosol and surface distribution of severe acute respiratory syndrome coronavirus 2 in hospital wards, Wuhan, China, 2020. Emerg Infect Dis 2020; 26(7):1583-1591

9 Alfa MJ, Olson N, Murray BL. Adenosine tri-phosphate (ATP)based cleaning monitoring in health care: how rapidly does environmental ATP deteriorate? J Hosp Infect 2015; 90(1):59-65

10 Osimani A, Garofalo C, Clementi F, Tavoletti S, Aquilanti L. Bioluminescence ATP monitoring for the routine assessment of food contact surface cleanliness in a university canteen. Int J Environ Res Public Health 2014; 11(10):10824-10837

11 Sanna T, Dallolio L, Raggi A, et al. ATP bioluminescence assay for evaluating cleaning practices in operating theatres: applicability and limitations. BMC Infect Dis 2018;18(1):583

12 Zemouri C, Volgenant CMC, Buijs MJ, et al. Dental aerosols: microbial composition and spatial distribution. J Oral Microbiol 2020;12(1):1762040

13 Ali AA, Altemimi AB, Alhelfi N, Ibrahim SA. Application of biosensors for detection of pathogenic food bacteria: a review. Biosensors (Basel) 2020;10(6):E58

14 Mulvey D, Redding P, Robertson C, et al. Finding a benchmark for monitoring hospital cleanliness. J Hosp Infect 2011;77(1):25-30

15 Volgenant CMC, Persoon IF, de Ruijter RAG, de Soet JJH. Infection control in dental health care during and after the SARS-CoV-2 outbreak. Oral Dis 2020;11

16 OBF. OBF. Available at: http://oralbiofilter.com. Accessed April 27,2020

17 Lloro V, Lozano-de Luaces V, Lloro I, Manzanares MC. The incidence of dental needs during isolated missions compared to non-isolated missions: a systematic review and implications for future prevention strategies. Mil Med 2019;184(3-4):e148-e155

18 Lloro Boada VA, Lloro Boada I. inventors; dispositivo auxiliar para procedimiento odontológico, método y usos del mismo. Spain patent application ES 2601486 B1. Available at: https:// patents.google.com/patent/ES2601486B1/fr. Accessed 2017

19 Eliades T, Koletsi D. Minimizing the aerosol-generating procedures in orthodontics in the era of a pandemic: current evidence on the reduction of hazardous effects for the treatment team and patients. Am J Orthod Dentofacial Orthop 2020;158(3):330-342

20 Koletsi D, Belibasakis GN, Eliades T. Interventions to reduce aerosolized microbes in dental practice: a systematic review with network meta-analysis of randomized controlled trials. J Dent Res 2020;99(11):1228-1238

21 Ho YH, Wang LS, Jiang HL, et al. Use of a sampling area-adjusted adenosine triphosphate bioluminescence assay based on digital image quantification to assess the cleanliness of hospital surfaces. Int J Environ Res Public Health 2016;13(6):E576

22 Bennett AM, Fulford MR, Walker JT, Bradshaw DJ, Martin MV, Marsh PD. Microbial aerosols in general dental practice. $\mathrm{Br}$ Dent J 2000;189(12):664-667 
23 Otter JA, Donskey C, Yezli S, Douthwaite S, Goldenberg SD, Weber DJ. Transmission of SARS and MERS coronaviruses and influenza virus in healthcare settings: the possible role of dry surface contamination. J Hosp Infect 2016;92(3):235-250

24 Hamid H, Khurshid Z, Adanir N, Zafar MS, Zohaib S. COVID19 pandemic and role of human saliva as a testing biofluid in point-of-care technology. Eur J Dent 2020;14(S 01):S123-S129

25 To KK, Tsang OT, Leung WS, et al. Temporal profiles of viral load in posterior oropharyngeal saliva samples and serum antibody responses during infection by SARS-CoV-2: an observational cohort study. Lancet Infect Dis 2020;20(5):565-574

26 Prather KA, Wang CC, Schooley RT. Reducing transmission of SARS-CoV-2. Science 2020;368(6498):1422-1424

27 Bizzoca ME, Campisi G, Muzio LL. Covid-19 pandemic: what changes for dentists and oral medicine experts? A narrative review and novel approaches to infection containment. Int J Environ Res Public Health 2020;17(11):E3793

28 Cook TM. Personal protective equipment during the coronavirus disease (COVID) 2019 pandemic - a narrative review. Anaesthesia 2020;75(7):920-927

29 Avasth A. High volume evacuator (HVE) in reducing aerosolan exploration worth by clinicians. J Dent Health Oral Disord Ther 2018;9(3):165-166

30 Desarda H, Gurav A, Dharmadhikari C, Shete A, Gaikwad S. Efficacy of high-volume evacuator in aerosol reduction: truth or myth? a clinical and microbiological study. J Dent Res Dent Clin Dent Prospect 2014;8(3):176-179

31 Lönnroth EC, Shahnavaz H. Users' demands regarding dental safety glasses. Combining a quantitative approach and grounded theory for the data analysis. Int J Occup Saf Ergon 2001;7(1):49-59

32 Chu DK, Akl EA, Duda S, Solo K, Yaacoub S, Schünemann HJ; COVID-19 Systematic Urgent Review Group Effort (SURGE) study authors. Physical distancing, face masks, and eye protection to prevent person-to-person transmission of SARS-CoV-2 and COVID-19: a systematic review and meta-analysis. Lancet 2020;395(10242):1973-1987

33 Farrier SL, Farrier JN, Gilmour AS. Eye safety in operative dentistry - a study in general dental practice. Br Dent J 2006;200(4):218-223, discussion 208

34 El-Boghdadly K, Wong DJN, Owen R, et al. Risks to healthcare workers following tracheal intubation of patients with COVID19: a prospective international multicentre cohort study. Anaesthesia 2020;75(11):1437-1447

$35 \mathrm{Wu} \mathrm{V}$, Noel CW, Forner D, et al. Considerations for head and neck oncology practices during the coronavirus disease 2019 (COVID-19) pandemic: Wuhan and Toronto experience. Head Neck 2020;42(6):1202-1208

36 Mick P, Murphy R. Aerosol-generating otolaryngology procedures and the need for enhanced PPE during the COVID-19 pandemic: a literature review. J Otolaryngol Head Neck Surg 2020;49(1):29

37 Herman JA, Urits I, Kaye AD, Urman RD, Viswanath O. COVID19: Anesthesia Management Recommendations. J Clin Anesth 2020. Doi: $10.1016 /$ j.jclinane.2020.109840

38 Dave M, Seoudi N, Coulthard P. Urgent dental care for patients during the COVID-19 pandemic. Lancet 2020;395(10232):1257

39 Farook FF, Mohamed Nuzaim MN, Taha Ababneh K, Alshammari A, Alkadi L. COVID-19 pandemic: oral health challenges and recommendations. Eur J Dent 2020;14(S 01):S165-S170

40 Ong SWX, Tan YK, Chia PY, et al. Air, surface environmental, and personal protective equipment contamination by severe acute respiratory syndrome coronavirus 2 (SARS-CoV-2) from a symptomatic patient. JAMA 2020;323(16):1610-1612 